\title{
Modelado matemático del secado de pastas libres de gluten en relación a la temperatura y humedad relativa del aire
}

\section{Mathematical modeling of the drying process of gluten-free pasta according to temperature and relative humidity}

\author{
Larrosa, Virginia (1), Lorenzo, Gabriel (2) (3), Zaritzky, Noemí (2) (3), Califano, Alicia (2) \\ (1) Facultad de Bromatología, Universidad Nacional de Entre Ríos, Argentina \\ (2)Centro de Investigación y Desarrollo en Criotecnología de Alimentos (CIDCA), CONICET-CICPBA, Facultad de \\ Ciencias Exactas, UNLP, La Plata, Argentina \\ (3) Departamento de Ingeniería Química, Facultad de Ingeniería, Universidad nacional de La Plata (UNLP), Argentina.
}

Contacto: lorenzogabriel@gmail.com

RECIBIDO: 26/2/2016 - APROBADO: 5/7/2016

\begin{abstract}
Resumen
El objetivo del trabajo fue presentar un modelo matemático que permita describir la cinética de secado de pastas libres de gluten (LG) y el efecto de variables operativas (temperatura y humedad del aire) sobre la velocidad del proceso, para lo cual se plantearon los balances de materia y energía acoplados utilizando propiedades de transporte dependientes del contenido de humedad y temperatura. Se consideraron pastas de dimensiones $200 \times 8,07 \times 1,80 \mathrm{~mm}$, de sección rectangular y transferencia bidireccional, y se adoptó un modelo difusional para el transporte de humedad en la pasta y condiciones de contorno convectivas para la transferencia de energía. Se realizaron ensayos para ajustar y validar el modelo, empleando cuatro condiciones de proceso, combinando temperaturas $\left(30^{\circ} \mathrm{C} \mathrm{y}\right.$ $50{ }^{\circ} \mathrm{C}$ ) y humedades relativas $(40 \%$ y $80 \%)$. Los resultados fueron modelados utilizando el método de elementos finitos para la resolución numérica de las ecuaciones, y se obtuvo un ajuste satisfactorio para todas las condiciones

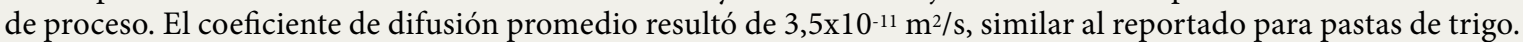
Se observó que las velocidades de secado obtenidas en las pastas LG fueron más altas que para pastas de trigo en iguales condiciones, lo cual puede atribuirse a la menor interacción del agua en la matriz LG.

Palabras clave: Modelado matemático, transferencia de energía, transferencia de materia.
\end{abstract}

\begin{abstract}
The aim of this work was to study the effect of operating variables (temperature and relative humidity (RH) of the air) on the drying rate of gluten-free (GF) pasta by mathematically modeling the drying process. The drying process was modeled using heat and mass coupled balances, considering bidirectional transport. A diffusional model for water transfer through pasta was assumed and heat transport was based on a conductive model. Pasta properties and transport coefficients were determined using pasta composition and process conditions. GF noodles geometry was $200 \times 8,07 \times 1,80 \mathrm{~mm}$ and a bidimensional transport was considered. Experimental runs were performed to validate the model. Four combinations of temperature $\left(30-50{ }^{\circ} \mathrm{C}\right)$ and relative humidity $(40-80 \%)$ were used, maintaining pasta dough composition constant. Experimental water losses were modeled using finite element method to solve the partial differential equations. The model fitting to experimental data was good and a validation with independent data was also satisfactory. The average water diffusional coefficient was $3,5 \times 10^{-11} \mathrm{~m}^{2} / \mathrm{s}$, similar to that reported for wheat pasta. GF noodles drying process was much faster than drying traditional wheat pasta under the same operating conditions.
\end{abstract}

Keywords: Mathematical modeling, heat transport, mass transport.

\section{Introducción}

El proceso de secado de las pastas supone un sistema compuesto por dos fases: el aire de secado y la masa. El equilibrio termodinámico se asume en la interfase de acuerdo con la isoterma de sorción, y la eliminación de agua se rige por un transporte simultáneo de calor y materia. El proceso generalmente se realiza exponiendo el material en forma continua al aire húmedo, proporcionado por ventiladores. El tiempo de secado depende de las características del equipo donde se 
realiza el proceso (temperatura de bulbo seco, humedad relativa (HR), velocidad del aire, etcétera) y de las características del producto (contenido de agua, relación volumen/superficie, etcétera). Durante el proceso la pasta desprende humedad con dificultad y lentitud a causa de fenómenos adsortivos y osmóticos. Por ello el proceso requiere un régimen específico que facilite el transporte de la humedad desde el interior hasta el exterior a través de los capilares y garantice un producto de buena calidad (Tscheuschner, 2001).

El secado de pastas de trigo tradicionales comienza con un producto con una humedad de $31 \%$ b.s. hasta aproximadamente un $13 \%$ b.s. y logra una mayor consistencia estructural otorgada por el retículo proteico que genera el gluten. Actualmente, el secado de la pasta en fábricas se realiza mediante el uso de aire caliente humedecido, con temperaturas en el rango entre 40 y $105^{\circ} \mathrm{C}$ y humedades relativas que varían entre el $40 \%$ y el $95 \%$ (De Temmerman, et al., 2007).

Cuando se trata de pastas aptas para celíacos, el proceso de secado se vuelve muy importante, dado que estas carecen de la red proteica que forma el gluten en las pastas tradicionales y que contiene a los gránulos de almidón dentro de la matriz. Un control inadecuado de la velocidad de secado puede conducir a pastas frágiles que se desintegran durante el proceso de cocción.

Este trabajo tiene como objetivo modelar numéricamente el proceso de transferencia simultánea de energía y materia que ocurre en la superficie de la pasta durante el secado de pastas libres de gluten aptas para celíacos, en contraposición al uso de soluciones analíticas más simples, que consideran únicamente la ley de Fick. Se estudiará el efecto de variables de proceso como la temperatura y humedad del aire de secado.

\section{Materiales y Métodos}

\section{Materiales}

Para la elaboración de la masa libre de gluten se utilizó almidón de maíz obtenido en Droguería Saporiti (Buenos Aires, Argentina); harina de maíz de Herboeste (Buenos Aires, Argentina) y huevo en polvo y clara de huevo en polvo provistas por OVOBRAND S.A. (Brandsen, Argentina). Los hidrocoloides utilizados fueron goma xántica y goma garrofín adquiridos a Sigma Chemical Co. (St. Louis, MO). Se empleó $\mathrm{NaCl}$ de grado analítico, aceite de girasol (Molinos Río de La Plata SACIFI, Buenos Aires), adquirido en un comercio local sin ningún tratamiento posterior, y se utilizó, además, agua destilada.

\section{Elaboración de la pasta}

Se prepararon pastas libres de gluten (LG), tipo cinta, utilizando una formulación y proceso de elaboración previamente optimizados por Larrosa et al. (2013), que contenía 51,4\% de almidón y harina de maíz, 6,6\% de proteínas de huevo en polvo (mezclas de huevo y clara secos, relación de 10:1), 2,5\% de mezcla de xántica/garrofín (relación 2:1), 36,0\% de agua, $2,5 \%$ de aceite y $1,0 \%$ de $\mathrm{NaCl}$. El porcentaje de concentraciones de la formulación se expresan en gramos/100 g total. Una vez obtenida la masa fue laminada hasta $2 \mathrm{~mm}$ de espesor aproximadamente. Finalmente se obtuvieron fideos LG frescos tipo cintas (dimensiones promedio: $20 \mathrm{~cm} \mathrm{x} \mathrm{8,07}$ $\mathrm{mm} \times 1,80 \mathrm{~mm}$ ), las cuales fueron secadas en las diferentes condiciones que se describen a continuación.

\section{Ensayos de secado}

El proceso se realizó en una estufa de aire forzado a velocidad constante, diseñada y construida ad hoc dentro del CIDCA, con sistemas independientes de control de humedad relativa del aire (sensor HIH4000A, Honeywell, USA) y de temperatura (termocuplas tipo "T") que permiten medir la pérdida de peso in situ de manera continua con una balanza ARC 120 (OHAUS, NJ, USA), como se muestra en la Figura 1. Se ensayaron cuatro condiciones de proceso diferentes combinando dos temperaturas $\left(30^{\circ} \mathrm{C}\right.$ y $\left.50^{\circ} \mathrm{C}\right)$ y dos humedades relativas ( 40 y $80 \%$ ), manteniendo constante la velocidad del aire en $0,5 \mathrm{~m} / \mathrm{s}$. Antes de comenzar cada ensayo, el equipo era colocado en funcionamiento un día antes con el objetivo de estabilizar la temperatura y humedad relativa a la cual sería secado el producto. El contenido de humedad de la pasta antes de comenzar y una vez finalizado el proceso de secado se determinó según el método AOAC 926.07 B (AOAC International, 2000).

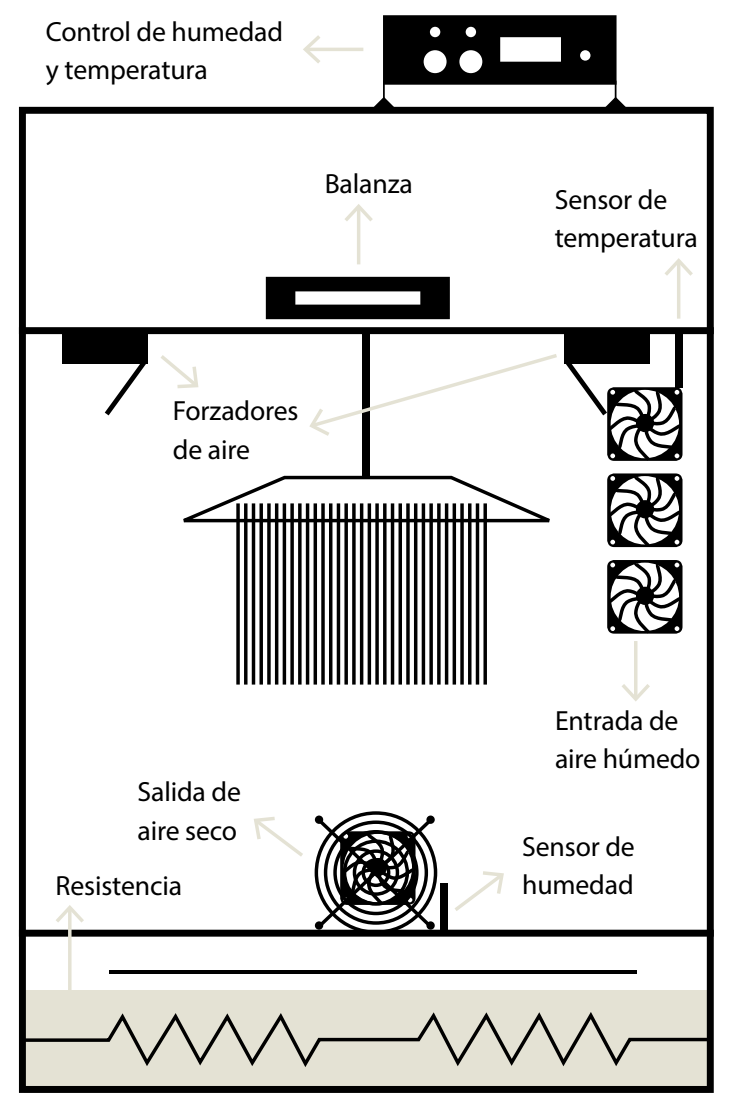

Figura 1. Esquema de la estufa de convección forzada con controles independientes de humedad y temperatura empleada en el secado de las pastas libres de gluten.

\section{Modelado matemático del proceso de secado}

El secado implica una transferencia simultánea de calor y materia. Es una operación básica donde intervienen dos factores importantes: la transmisión de calor, para suministrar 
el calor latente de evaporación del agua, y la transferencia de agua, que implica su movimiento a través de los productos alimenticios y su separación de los mismos.

El proceso de secado presenta generalmente un breve período de velocidad constante y posteriormente se produce un período de velocidad decreciente, donde el factor que controla es la difusión. Asumiendo para la etapa difusional de transporte de humedad a través de la pasta un modelo basado en la segunda ley de Fick en estado no estacionario resulta:

$$
\frac{\partial C}{\partial t}=\boldsymbol{\nabla} \cdot(D \nabla C)
$$

donde $C$ es la concentración de agua en el sólido (mol de agua $/ \mathrm{m}^{3}$ de masa) y $\mathrm{D}$ el coeficiente de difusión efectivo $\left(\mathrm{m}^{2} / \mathrm{s}\right)$.

Por otro lado, la transferencia de energía en el sistema está dada por:

$$
\rho C_{P} \frac{\partial T}{\partial t}=\boldsymbol{\nabla} \cdot(k \boldsymbol{\nabla} T)
$$

donde $\rho, C_{P}$ y $k$ son la densidad, calor específico y conductividad térmica del sólido, respectivamente.

Por medio de las condiciones de contorno descriptas en las Ecuaciones (3) y (4), las ecuaciones diferenciales no estacionarias de transferencia de materia y energía (Ecuaciones (1) y (2)) quedan acopladas, considerando además las condiciones de simetría tanto para el balance de energía como para el de materia en el centro de la pasta $(\mathrm{x}=0$, $\mathrm{y}=0$ ). La Ecuación (3) relaciona la energía transportada por convección desde el aire hasta la superficie de la pasta con el aumento de calor sensible en el sólido y el calor latente requerido para la evaporación del agua; mientras que la Ecuación (4) vincula el flujo difusivo de agua dentro del sólido con el flujo convectivo de agua en el aire.

En las interfases pasta/aire se cumple que:

$$
\begin{aligned}
& h\left(T_{\infty}-T_{s}\right)=-\boldsymbol{n} \cdot(-k \nabla T)+\lambda k_{m}\left(C_{i}^{\prime}-C_{b}^{\prime}\right) \\
& -\boldsymbol{n} \cdot(\rho D \nabla C)=k_{m}\left(C_{i}^{\prime}-C_{b}^{\prime}\right)=\frac{k_{m}}{m}\left(C_{i}-C_{b}\right)
\end{aligned}
$$

donde $h$ es el coeficiente de transferencia calórica $\left(\mathrm{W} / \mathrm{m}^{2}{ }^{\circ} \mathrm{C}\right)$, $T_{\infty}$ es la temperatura del aire de secado, $T_{s}$ la temperatura en la interfase del sólido, $\lambda$ el calor latente de evaporación del agua, $k_{m}$ es el coeficiente de transferencia de materia, $m$ es la pendiente de la curva de equilibrio (isoterma de sorción), $C_{i}{ }_{i}$ y $C_{b}{ }_{b}$ son las concentraciones de agua en la interfase y en el seno del aire, respectivamente evaluadas por unidad de volumen de fase gaseosa, y $C_{i}$ y $C_{b}$ las mismas concentraciones evaluadas por volumen de sólido. En la Figura 2 se muestran esquemáticamente los perfiles de humedad y temperatura dentro de la pasta y en el aire de secado.

Se utilizó el método de elementos finitos para la resolución de las ecuaciones diferenciales a derivadas parciales mediante el software Comsol Multiphysics. Para las simulaciones se consideraron las siguientes suposiciones: 1) Un sistema de transporte bidimensional;2) distribución inicial de agua uniforme dentro de la pasta; 3 ) las dimensiones de la pasta no cambiaron durante el secado; 4) se considera que el sólido es homogéneo e isotrópico. Para cada condición de proceso se alimentaron las propiedades físicas del sólido y del fluido calefactor (De Temmerman, et al., 2007; Migliori, et al., 2005; Veladat, et al., 2012).

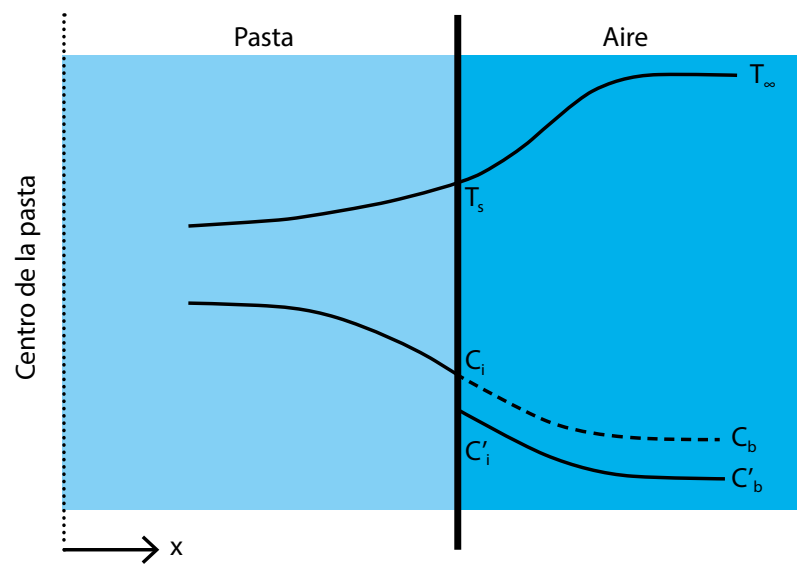

Figura 2. Esquema de la interfase aire de secado-pasta junto con los perfiles de temperatura y concentración de agua.

\section{Resultados y Discusión}

\section{Determinación de las curvas de secado}

Las curvas experimentales (Figura 3) muestran la disminución de la humedad de los fideos desde su contenido inicial promedio de $0,65 \mathrm{~g}$ de agua/g b.s. hasta el valor deseado de $0,13 \mathrm{~g}$ de agua/g b.s, que fueron determinadas por medio de la pérdida de peso durante el secado. Se consideró como punto final del proceso cuando la actividad acuosa de la pasta estaba por debajo de 0,65 (Kill y Turnbull, 2001), y a partir de las isotermas de sorción se estableció el contenido de humedad en equilibrio con dicha $\mathrm{a}_{\mathrm{w}}$. En todas las curvas se puede observar que inicialmente, y por un breve período, el contenido de agua disminuyó linealmente y luego comenzó a disminuir más lentamente, entrando en el período de velocidad decreciente.

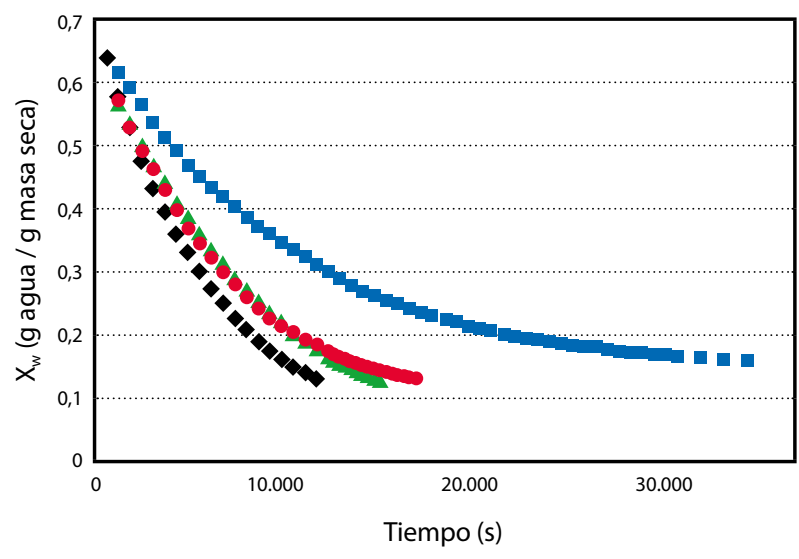

Figura 3. Curvas de secado experimentales para las distintas condiciones de Temperatura (T) y humedad relativa (HR) del aire de secado. $(\Delta) \mathrm{T}=30^{\circ} \mathrm{C}, \mathrm{HR}=40 \%$; $(\bullet) \mathrm{T}=30^{\circ} \mathrm{C}, \mathrm{HR}=80 \%$; $(\bullet) \mathrm{T}=50{ }^{\circ} \mathrm{C}, \mathrm{HR}=40 \%$; $\bullet$ ) $\mathrm{T}=50{ }^{\circ} \mathrm{C}, \mathrm{HR}=80 \%$.

El tiempo de secado de las pastas libres de gluten varió entre los 190 y 560 minutos (3,2-9,3 horas); el proceso más largo fue el correspondiente a la condición de baja temperatura y alta humedad relativa (Tabla 1). Por el contrario, el tiempo 
de secado se redujo significativamente cuando se utilizó la condición de alta temperatura y baja humedad relativa. Estos valores son más cortos que los tiempos de fideos de trigo secados aproximadamente a $60^{\circ} \mathrm{C}$ (entre 14 y 20 horas) y similares a los tiempos de secado a más altas temperaturas $\left(80-120^{\circ} \mathrm{C}\right)$, con 3 a 8 horas de proceso (Kill y Turnbull, 2001).

\begin{tabular}{|c|c|c|c|}
\hline \multicolumn{2}{|c|}{} & \multicolumn{2}{|c|}{ Temperatura de secado $\left({ }^{\circ} \mathrm{C}\right)$} \\
\cline { 3 - 4 } \multicolumn{2}{|c|}{} & 30 & 50 \\
\hline \multirow{3}{*}{ HR (\%) } & 40 & 4,00 & 3,17 \\
& 80 & 9,33 & 4,58 \\
\hline
\end{tabular}

Tabla 1. Tiempos de secado promedio (en h) de pastas libres de gluten secadas en distintas condiciones de temperatura y humedad relativa (HR) del aire de secado.

\section{Cálculo de las propiedades termofísicas y coeficientes de transferencia}

Para el cálculo del calor específico $\left(C_{\mathrm{p}}\right)$ del sólido se utilizó el promedio pesado de los $C_{\mathrm{p}}$ de sus componentes mediante sus fracciones másicas, $w_{\mathrm{i}}$ (Migliori, et al., 2005):

$C_{p}=\left[\frac{X}{1+X} C_{p, a g u a}+\frac{1}{1+X} C_{p, s o l}\right]$

$C_{p, \text { sol }}=w_{\text {almidón }} C_{p, \text { almidón }}+w_{\text {proteína }} C_{p, \text { proteína }}$

$+w_{\text {grasa }} C_{p, \text { grasa }}$

$C_{p, \text { almidón }}=5.737(T)+1328 \quad[\mathrm{~J} /(\mathrm{kg} \mathrm{K})]$

$C_{p, \text { proteína }}=6.329(T)+1465 \quad[J /(k g K)]$

$C_{p, \text { grasa }}=2000 \quad[\mathrm{~J} /(\mathrm{kg} \mathrm{K})]$

Para la conductividad térmica de la pasta se adoptó el modelo propuesto por Saravacos y Maroulis (2001):

$k(X, T)=\frac{\lambda_{0}}{1+X} \exp \left[-\frac{E_{0}}{R}\left(\frac{1}{T}-\frac{1}{T_{\text {ref }}}\right)\right]$
$+\frac{X \lambda_{i}}{1+X} \exp \left[-\frac{E_{i}}{R}\left(\frac{1}{T}-\frac{1}{T_{\text {ref }}}\right)\right]$

donde $R$ es la constante de gases ideales, $\lambda_{0}(0.273 \mathrm{~W} / \mathrm{mK})$ y $\lambda_{i}(0.8 \mathrm{~W} / \mathrm{mK})$ las conductividades térmicas a $T_{\text {ref }}=60^{\circ} \mathrm{C} \mathrm{y}$ humedades $\mathrm{X}=0$ y X $=\infty$, respectivamente; $E_{0}$ y $E_{i}$ las energías de activación para conducción de calor para $\mathrm{X}=0$ y $\mathrm{X}=\infty$, respectivamente.

La densidad del sólido se halló experimentalmente determinando el peso y volumen de diferentes muestras de pasta. Se tomaron al menos 10 especímenes de cada condición de secado y se determinaron sus dimensiones mediante un calibre digital (modelo SC111201, Schwyz S.A) y su peso mediante balanza analítica. En base a estos valores se estableció la densidad de cada fideo y se obtuvo su valor promedio para introducirlo en el modelo.

El coeficiente de difusión del agua en la pasta fue el único coeficiente que se ajustó utilizando los datos experimentales y se validó a través de experimentos independientes, por medio de combinaciones temperatura/ humedad relativa, comparando las pérdidas de peso predichas por el modelo con las obtenidas.
El coeficiente de transferencia de energía (h) se calculó mediante correlaciones del tipo $\mathrm{Nu}=\mathrm{f}(\mathrm{Re}, \mathrm{Pr})$ para flujo alrededor de objetos sumergidos, placa plana y en régimen laminar $\operatorname{Re}<3 \times 10^{5}$ (Geankoplis, 2003).

$N u=0.648 R e^{0.5} \operatorname{Pr}^{1 / 3}$

donde $N u=\frac{\mathrm{hL}}{\mathrm{k}}, R e=\frac{\rho \mathrm{vL}}{\mathrm{y}} \operatorname{Pr}=\frac{\mathrm{C}_{\mathrm{P}} \mu}{\mathrm{k}}$ son los números de Nusselt, Reynolds y Prandtl, respectivamente, determinados a partir de las propiedades físicas del aire en cada condición de proceso específica y L es la longitud característica (se adoptó $\mathrm{L}=8,07 \mathrm{~mm}$ para este caso).

Para el cálculo del coeficiente de transferencia de materia $\left(\mathrm{k}_{\mathrm{m}}\right)$ se utilizó la analogía entre transferencia de energía y materia (De Temmerman, et al., 2007):

$\frac{h}{k_{m}}=\rho C_{P}\left(\frac{\alpha}{D_{\text {agua }}}\right)^{2 / 3}$

donde a es la difusividad térmica del aire y $D_{\text {agua }}$ es el coeficiente de difusión de agua en aire a cada temperatura.

\section{Resultados del modelo}

En la Figura 4a se muestra, a modo de ejemplo, el satisfactorio ajuste del modelo a los datos experimentales correspondientes a pastas libres de gluten, para un secado a $50^{\circ} \mathrm{C}$ y humedad relativa del aire de $80 \%$. Las diferentes condiciones operativas presentaron un comportamiento análogo con un error porcentual promedio en el ajuste inferior al 5\%. Como único parámetro de ajuste se utilizó el coeficiente de difusión, cuyo valor promedio resultó de $3,5 \times 10^{-11} \mathrm{~m}^{2} / \mathrm{s}$ similar al reportado por otros autores para pastas de trigo tradicionales (Mercier, et al., 2013).

El uso de soluciones analíticas más simples aborda el problema considerando únicamente la ley de Fick, sin acoplar el proceso de transferencia de energía y la evaporación que ocurre en la superficie de la pasta. Además, se considera que el secado se realiza en condiciones isotérmicas, en las cuales el sólido alcanza instantáneamente la temperatura del aire, sin tener en cuenta la existencia del coeficiente $h$. Sin embargo, puede observarse a partir del modelo numérico (Figura 4c) que la temperatura del centro alcanza su valor asintótico recién después de aproximadamente $30 \mathrm{~min}$. Asimismo, las soluciones analíticas de difusión pura consideran que la concentración de agua en la interfase se mantiene constante, sin contemplar la resistencia a la transferencia de materia del lado del aire, expresada a partir del coeficiente de transferencia de materia.

Los resultados obtenidos se compararon con el secado de pastas tradicionales formuladas con harina de trigo, bajo las mismas condiciones de humedad y temperatura. Se observó que el tiempo de proceso de las pastas libres de gluten resultó al menos un 50\% inferior que en las pastas de harina de trigo, lo cual probablemente puede estar asociado a la alta capacidad de retención de agua de las proteínas del trigo.

\section{Conclusión}

El tiempo experimental de secado de las pastas libres de gluten resultó muy dependiente de las condiciones operativas, variando entre 190 y 560 minutos para llegar a una humedad final de $0,13 \mathrm{~g} / \mathrm{g}$. Los tiempos de secado fueron similares a los de pastas de trigo secadas a temperaturas mucho más altas. 

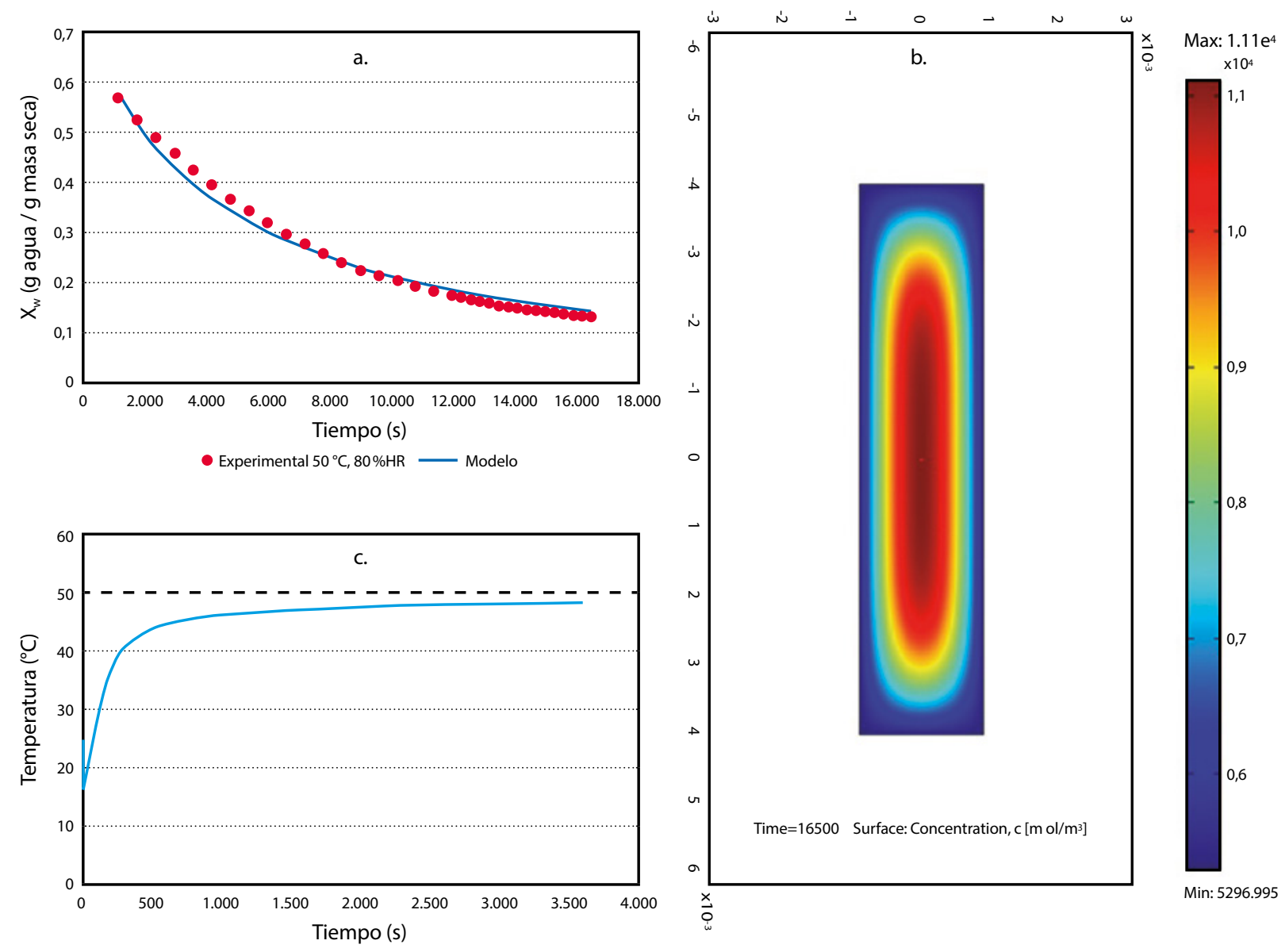

Figura 4. (a) Ajuste del modelo a los datos experimentales para las condiciones $50{ }^{\circ} \mathrm{C}$ y $80 \%$ humedad del aire (propiedades usadas: $\left.k=0,48 \mathrm{~W} /(\mathrm{mK}) ; h=29,5 \mathrm{~W} /\left(\mathrm{m}^{2} \mathrm{~K}\right) ; k_{m}=2,95 \times 10^{-2} \mathrm{~m} / \mathrm{s} ; C_{b}=5297 \mathrm{~mol} / \mathrm{m}^{3} ; D=3,5 \times 10^{-11} \mathrm{~m}^{2} / \mathrm{s}\right)$. (b) Distribución de la concentración de agua dentro de la pasta $\left(\mathrm{mol} / \mathrm{m}^{3}\right)$ luego de $275 \mathrm{~min}$ de secado. (c) Historia térmica del centro de la pasta $\left(\mathrm{T}_{\text {inicial }}=25^{\circ} \mathrm{C}, \mathrm{T}_{\infty}=50^{\circ} \mathrm{C}\right)$ durante los primeros $60 \mathrm{~min}$ de proceso.

En este trabajo se modelaron adecuadamente las curvas de secado ajustando únicamente el coeficiente de difusión, cuyo valor promedio resultó similar al observado en pastas de trigo tradicionales. El modelado matemático resulta una herramienta adecuada para predecir tiempos de proceso y analizar el efecto de parámetros como temperatura, velocidad, humedad del aire, etcétera, en los tiempos de secado.

\section{Reconocimientos}

Los autores agradecen el financiamiento del Consejo Nacional de Investigaciones Científicas y Técnicas (CONICET) y la Universidad Nacional de La Plata (UNLP). Los autores agradecen también la colaboración de OVOBRAND S.A., Brandsen, Argentina.

\section{Referencias}

AOAC International, 2000. Official Methods of Analysis of AOAC International. 13a ed. Gaithersburg: AOAC. Official Method 926.07 B.

De Temmerman, J., Verboven, P., Nicolai, B. y Ramon, H., 2007. Modelling of transient moisture concentration of semolina pasta during air drying. En: Journal of Food
Engineering, 80(3), pp.892-903.

Geankoplis, C., 2003. Transport processes and separation process principles (includes unit operations). 3ra ed. New Jersey: Prentice Hall Press.

Kill, R.C y Turnbull, K., 2001. Pasta and semolina technology. Oxford: Blackwell Science Ltd.

Larrosa, V., Lorenzo, G., Zaritzky, N. y Califano, A., 2013. Optimization of rheological properties of gluten-free pasta dough using mixture design. En: Journal of Cereal Science, 57(3), pp.520-526.

Mercier, S., Moresoli, C., Villeneuve, S., Mondor, M. y Marcos, B., 2013. Sensitivity analysis of parameters affecting the drying behaviour of durum wheat pasta. En: Journal of Food Engineering, 118(1), pp.108-116.

Migliori, M., Gabriele, D., de Cindio, B. y Pollini, C.M., 2005. Modelling of high quality pasta drying: mathematical model and validation. En: Journal of Food Engineering, 69(4), pp.387-397.

Saravacos, G. D. y Maroulis, Z. B., 2001. Transport properties of foods. New York: CRC Press.

Tscheuschner, H.D., 2001. Fundamentos de tecnología de los alimentos. Zaragoza: Ed. Acriba S.A.

Veladat, R., Zokaee Ashtiani, F., Rahmani, M. y Miri, T., 2012. Review of numerical modeling of pasta drying, a closer look into model parameters. En: Asia-Pacific Journal of Chemical Engineering, 7(2), pp.159-170. 